\title{
О СУБОПТИМАЛЬНОЙ СТАБИЛИЗАЦИИ ДИНАМИЧЕСКИХ СИСТЕМ СПОСОБОМ ОБРАЩЕНИЯ И АГРЕГАЦИИ
}

\author{
(Представлена Н. Алумяэ)
}

Для управляемой динамической системы предложены два критерия стабилизации. Методом пронзводящих функций и агрегирующих переменных $y$ доказана теорема о субоптимальном синтезе $u(t, x, a)$. Получены условия минимизации показателя для выбора $a$. Приводится пример.

1. Рассмотрим уравнения возмущений $x \equiv 0$ управляемой системы

$$
\begin{aligned}
& x=F(t, x, u) t, x \in R=\{t \geqslant 0,|x|<\infty\}, F(t, 0,0) \equiv 0, F \subset C_{1}(R \times \Omega), \\
& u=\left(u_{\sigma}\right)^{*} \in U^{0}=\left\{u \in \Omega \subseteq E^{s}, u \in C_{1}\left(R^{\prime}\right)\right\}, R^{\prime}=R_{0} \backslash x^{\prime}=0, R_{0}=R \backslash\left|x^{1}\right|>H_{0}, \\
& x=\left(x_{v}, x_{h}\right)^{*}, x^{1}=\left(x_{v}\right)^{*}, x^{(2)}=\left(x_{h}\right)^{*}, v=\overline{1, m_{1}} \leqslant \operatorname{dim} x=n, h=\overline{m_{1}+1, n}, \\
& x^{1}=\left(x_{\alpha}, x_{\beta}\right)^{*}, x^{\prime}=\left(x_{\alpha}\right)^{*}, x^{\prime \prime}=\left(x_{\beta}\right)^{*}, \alpha=\overline{1, m_{0}} \leqslant m_{1}, \beta=\overline{m_{0}+1, m_{1}}
\end{aligned}
$$

с компактом $\Omega$, целью $Q=\left\{x \mid x^{\prime}=0\right\}$ и критерием

$$
J(t, x \mid u)=\int_{t}^{t_{1}} F_{0}(\tau, x, u) d \tau \rightarrow \min _{u}\left(\lim x^{\prime}=0, t \rightarrow t_{1}-0, F_{0} \in C_{1}\right),
$$

где $F, U^{0}$ удовлетворяют условиям существования, единственности и продолжаемости $x[t]$ на $t \in\left[t_{0}, t_{1}\right), x \in R^{\prime}, t_{1}$ - первый момент примыкания $x$ к $Q$ в (1.2). Регулятор $u^{\prime} \in U^{0}-x^{1}, x^{\prime}$-стабилизирующий (1.1), если имеем $\left|x^{1}\right| \leqslant \varepsilon^{\prime} \leqslant H_{0}\left(t \in\left[t_{0}, t_{1}\right]\right), \quad x^{\prime}[t] \rightarrow 0$ при $t \rightarrow t_{1}-0$, $\forall\left|x_{0}^{\prime}\right| \leqslant \delta_{0}^{\prime} \leqslant \delta_{0}{ }^{1}=$ const, где $\forall \varepsilon>0$, фиксируются $t_{0} \geqslant 0, \quad\left|x_{0}{ }^{(2)}\right|<\infty$, $H_{0}=$ const $>0$ и существует $\delta_{0}{ }^{1}=\delta_{0}{ }^{1}\left(t_{0}, x_{0}{ }^{(2)}, \varepsilon^{\prime}\right) \geqslant\left|x_{0}{ }^{1}\right|>0$. Используются $\left[{ }^{1}\right]$ обозначения $x_{0}=x\left[t_{0}\right], x_{0}^{\prime} \neq 0,\left|x_{0}^{\prime \prime}\right|<\delta_{0}^{1}, x[t]=x\left(t, t_{0}, x_{0} \mid u^{\prime}\right)$. Допустимый $u^{0}$ из класса $\left\{u^{\prime}\right\} \subseteq U^{0}-$ оптимальный для (1.1) по (1.2), если $J\left(t, x \mid u^{0}\right) \leqslant J\left(t, x \mid u^{\prime}\right)$ при $\left|x_{0}{ }^{1}\right| \leqslant \delta_{0}^{1}, 0<\left|x_{0}^{\prime}\right| \leqslant \delta_{0}^{\prime},\left(t_{0}, x_{h 0}{ }^{(2)}, H_{0}\right)^{*}=$ $=$ fixconst. Введем для стабилизации и наблюдаемости переменные

$$
\begin{aligned}
& y=y(t, x, a)=\left(y_{i}\right)^{*}=\left(y_{j}^{\prime}, y_{d}^{\prime \prime}\right)^{*}\left(i, l=\overline{1, l_{0}} \leqslant n, j=\overline{1, l_{1}}, d=l_{1}+1, l_{0}\right), \\
& y^{\prime}=\left(y_{j}^{\prime}\right)^{*}, y^{\prime \prime}=\left(y_{d}^{\prime \prime}\right)^{*},\left.y\right|_{x^{\prime}=0} \equiv 0,\left.y^{\prime}\right|_{x^{\prime}=0} \equiv 0 \quad\left(r^{1}=\left|x^{1}\right|, \quad r^{\prime}=\left|x^{\prime}\right|, \quad l_{0} \ll n\right) \text {, } \\
& \text { rank }\left\|\partial y_{i} / \partial x_{l}\right\|=l_{0}, y \in C\left(R_{0} \times A\right), y \in C_{1}\left(R^{\prime} \times A\right), \quad|y|=\mathrm{Q}_{0} \geqslant \mathrm{Q}_{1} \quad\left(r^{1}, a\right) \gg 0 \text {, } \\
& \left|y^{\prime}\right|=\mathrm{Q}^{\prime} \geqslant \mathrm{Q}_{1}\left(r^{\prime}, a\right) \gg 0, \quad \mathrm{Q}_{1}\left(\xi_{2}, a\right) \geqslant \mathrm{Q}_{1}\left(\xi_{1}, a\right), \quad \mathrm{Q}_{1}^{\prime}\left(\xi_{2}, a\right) \geqslant \mathrm{Q}_{1}^{\prime}\left(\xi_{1}, a\right)\left(\xi_{2}>\xi_{1}\right) \text {, } \\
& \mathrm{Q}_{1}(0, a)=\mathrm{Q}^{\prime}{ }_{1}(0, a)=0, \mathrm{Q}_{1}(\xi, a) \geqslant \mathrm{Q}^{\prime}{ }_{1}(\xi, a)>0, \xi>0, \mathrm{Q}_{1}, \mathrm{Q}^{\prime}{ }_{1} \in C\left(R_{0} \times A\right) \text {, }
\end{aligned}
$$


где агрегаты $y_{i}$ - вообще нелинейные по $x, a$ связки Четаева с вектором $\left[{ }^{2,3}\right]$ агрегации $a=\left(a_{m}\right)^{*}$ из области $A \subseteq E^{N}, a=$ const, $m=\overline{1, N}$.

В силу (1.3) для $\forall h \leqslant \varrho_{1}(H, a)=\max \varrho_{1}\left(\left|x^{1}\right| \leqslant H_{0}\right)|y| \leqslant h$ отвечает прообраз в $\left|x^{1}\right| \leqslant \delta\left(\varrho_{1}(\delta, a)=h_{\delta}\right)$, из $\left|y^{\prime}\right| \rightarrow 0$ следует $\left|x^{\prime}\right| \rightarrow 0$. Для $\forall\left(t, x_{h}\right)^{*}, \quad a=$ fixconst $\in A$ область $\{y\}$ содержит $|y| \leqslant h$. Поэтому для оптимальной $x^{1}, x^{\prime}$-стабилизации (1.1), (1.2) достаточно решить в переменных $\xi$ задачу оптимизации

$\xi=f(t, \xi, u, a), \xi \in D^{\prime}=\xi\left(R^{\prime}\right), f(t, 0,0, a) \equiv 0 \quad\left(D^{\prime}=D \backslash y^{\prime}=0\right)$,

$y=Y(t, \xi, u, a), \gamma^{*}=\Gamma(t, \xi, u, a) \quad\left(\gamma_{k} \equiv x_{k}, x_{l}=\eta_{l}(t, \xi, a) \in C_{1}\left(D^{\prime} \times A\right)\right)$,

$Y=\partial y / \partial t+F \cdot \partial y / \partial x, \Gamma=\left(F_{k}\right)^{*} \mid x_{l}=\eta_{l} \quad\left(l=\overline{1, l_{0}}, k=\overline{l_{0}+1, n}\right)$,

$I=\int_{t}^{t_{1}} f_{0} d \tau \rightarrow \min _{u}, f_{0}=\left.F\right|_{x=x(t, \xi, a)} \in C_{1}\left(D^{\prime} \times \Omega \times A\right)$

при условии $y, y^{\prime}$-стабилизации (1.4) $I$-оптимальным $u^{1}(t, \xi, a)$, где $|y| \leqslant h \leqslant H \quad\left(t_{0} \leqslant t \leqslant t_{1}\right), \quad \lim y^{\prime}=0 \quad$ при $\quad t \rightarrow t_{1}-0 \quad\left(\forall\left|y_{0}\right| \leqslant \delta\right.$, $H=$ fixconst). Пусть (1.4) имеет в $U^{0}$ подкласс возможных $u^{\prime}$, порожденных $V(t, \xi, a)$ вида

$V(t, \xi, a) \geqslant V_{1}(\varrho, a)>\left.V\right|_{y=0} \equiv 0, \varrho \neq 0 \quad\left(0 \ll V_{1} \in C([0, H] \times A)\right)$,

$-\left.V^{\cdot}\right|_{u=u^{\prime}}=W(t, \xi, a) \geqslant 0, y^{\prime} \neq 0,|y| \equiv \varrho \leqslant H, \forall a \in A, \xi \in D_{0}^{\prime}$,

$D_{0}=D \cap\{|y| \leqslant H\}, D^{\prime}{ }_{0}=D^{\backslash} y^{\prime}=0, D=\xi\left(R_{0}\right), t \geqslant 0, W \subset C\left(D^{\prime}{ }_{0} \times A\right)$.

Из (1.3), (1.5) для (1.4) при $u=u^{\prime} \in U^{0}$ имеем $y, y^{\prime}$-устойчивость

$\forall \varepsilon \leqslant H \quad \exists \delta=\delta\left(\varepsilon, t_{0}, \gamma_{0}, a \mid V\right):|y| \leqslant \varepsilon\left(t \in\left[t_{0}, t_{1}\right), \forall\left|y_{0}\right| \leqslant \delta \leqslant H\right)$,

$\forall \varepsilon \leqslant H \exists \delta^{\prime}=\left[\delta^{2}-\left|y_{0}^{\prime \prime}\right|^{2}\right]^{1 / 2} \leqslant \delta:\left|y^{\prime}\right| \equiv r \leqslant \varepsilon\left(\delta^{0} \equiv \delta(H), \delta^{\prime 0}=\delta^{\prime}(H)\right)$.

Остальные свойства $V, U^{\prime}, f\left(u^{\prime}\right)$, достаточные для $y^{\prime} \rightarrow 0$ при $t \rightarrow t_{1}-0$ и фиксированных $t_{0}, y_{0}^{\prime \prime}, \gamma_{0}, \delta^{0}, \delta^{\prime 0}$, доопределим ниже. Допустим противное: в области $D_{0}^{\prime}(1.5)$ системы (1.4) есть $\xi *[t]$ с $y *^{\prime}[t]$-компонентой, не сходящейся к нулю при $r_{0}=r\left[t_{0}\right] \leqslant \delta^{\prime}$ и $\forall t_{1} \leqslant+\infty\left(r_{0}>0\right.$, $\left.t_{1}>t_{0}\right)$. Из (1.6) следует, что $r * \equiv|y *|$ удовлетворяет условиям, задающим лишь два класса $(1.7),(1.8)\{r \cdot[t]\}$ функций вида $C *^{1}, C \cdot{ }^{(2)}$

$\lim r *=l^{0} \leqslant H\left(t \rightarrow+\infty, 0<l^{0}\right), \exists \tau_{n} \rightarrow+\infty, r_{* n} \rightarrow l^{0}: r_{*} *_{n} \rightarrow 0$,

$r_{* n}=r_{*}\left[\tau_{n}\right], r_{* n}=d r_{*} / d t \mid t=\tau_{n}, \quad 0<r_{*}[t] \subset C_{1}\left[t_{0}, \infty\right), C_{*}^{1}=\left\{r_{*}[t]\right\}$,

$\underline{\lim } r_{*}[t]=l^{1}<l^{(2)}=\varlimsup_{\lim } r_{*}[t]\left(t \rightarrow+\infty, 0 \leqslant l^{1}<l^{(2)} \leqslant H\right)$

$$
\left(0<r * \subset C_{1}\left[t_{0}, \infty\right)\right) \text {, }
$$

$\exists \tau_{k}^{\prime} \rightarrow+\infty, r_{*_{k}}^{\prime} \rightarrow l^{1}: r_{*_{k}}^{\prime}=0\left(r_{*_{k}}^{\prime}=r_{*}\left[\tau_{k}^{\prime}\right], \tau_{k}^{\prime}<\tau_{k}^{\prime \prime}<\tau_{k+1}^{\prime}<\tau_{k+1}^{\prime \prime}\right)$,

$\exists \tau_{k}^{\prime \prime} \rightarrow+\infty, r_{*_{k}}^{\prime \prime} \rightarrow l(2): r_{* k}^{\prime \prime}=0\left(r_{* k}^{\prime \prime}=r_{*}\left[\tau_{k}^{\prime \prime}\right], n, k=\overline{1, \infty}, C(2)=\left\{r_{*}[t]\right\}\right)$,

$\forall l^{*} \in\left(l^{1}, l(2)\right) \exists \tau_{k}^{*} \rightarrow+\infty: r_{*}\left[\tau_{k}^{*}\right]=l^{*}, r_{*}[t] \rightarrow\left[l^{1}, l(2)\right](t \rightarrow f \infty)$,

$\forall \varepsilon_{1}, \varepsilon_{2} \exists T\left(\varepsilon_{1}, \varepsilon_{2}\right): l^{1}-\varepsilon_{1}<r \cdot[t]<l(2)+\varepsilon_{2}, t \geqslant T \geqslant t_{0} \geqslant 0$.

Для $\forall r .[t] \in C .{ }^{(2)} \quad$ имеем последовательности $\left(\tau^{\prime}{ }_{k}, r^{\prime}{ }_{*}\right) \rightarrow\left(\infty, l^{1}\right)$, $\left(\tau_{k}^{\prime \prime}, r_{*}{ }^{\prime \prime}\right) \rightarrow\left(\infty, l^{(2)}\right)\left(l^{1}<l^{(2)}\right)$ стационарных $r_{*}^{*}=0$ точек $r \cdot[t]-$ «экстремумов» ее колебаний, В классе $C_{\text {.(1) }}^{(1)}$ возможны постоянные и моно- 
тонные функции, которых класс $C .{ }^{(2)}$ не имеет. Функция $r[t] \equiv C{ }^{1} \cup C .{ }^{(2)}$, если нет $\left(t_{n}, r_{n}\right)^{*} \rightarrow(+\infty, l)^{*}$ с $r_{n}{ }^{*} \rightarrow 0\left(l>0, r_{n}=r\left[t_{n}\right]\right)$.

Введем совокупность $P=\left\{\left(\infty, r^{*}\right)^{*}\right\} \subset \bar{D}_{0}^{\prime}\left(0<r^{*} \leqslant H\right)$ предельных для $p_{m}{ }^{*}=\left(t_{m}{ }^{*}, r_{m}{ }^{*}\right)^{*}$ точек со свойствами: $t_{m}{ }^{*} \rightarrow \infty, r_{m}{ }^{*} \rightarrow r^{*}$, $R_{m}{ }^{*} \rightarrow 0, \quad m \rightarrow \infty, \quad$ где $r_{m}{ }^{*}=\left|y_{m}{ }^{*}\right|, \quad R=r, R_{m}{ }^{*}=R\left(t_{m}{ }^{*}, \xi_{m}{ }^{*}, a \mid u^{\prime}\right)$. $P-$ множество $r$-агрегированных и предельных к $t_{m}{ }^{*} \rightarrow+\infty$, $\xi_{m}{ }^{*}\left(r_{m}{ }^{*} \rightarrow r^{*}>0\right)$ точек в $D_{0}^{\prime}$, на которых $r_{m} \rightarrow 0$ при $m \rightarrow+\infty$. Из (1.7), (1.8) следует, что $P$ непусто, если на $D_{0}^{\prime}$ имеем $\left\{r_{*}[t]\right\} \neq \varnothing$. $P \cup 0-$ замкнуто. При $t_{m} \rightarrow+\infty, r_{m} \rightarrow r \in[0, H]$ вообще $R_{m} \nrightarrow 0$. Но для элементов $p_{n}, p_{n}{ }^{\prime}, p_{n}{ }^{\prime \prime}$ имеем

$$
R_{n}=r_{n} \rightarrow 0 \text { при } t_{n}=\tau_{n}, \tau_{n}^{\prime}, \tau_{n}^{\prime \prime}, r_{n}=r_{*_{n}}, r_{*_{n}}^{\prime}, r_{*_{n}}^{\prime \prime}\left(r_{n} \rightarrow r\right) \text {, }
$$

ибо их пределы лежат в $P \cup 0$. Пусть для $\forall r^{*} \in(0, H]$ существуют $\varepsilon^{*}=\varepsilon_{*}\left(r^{*}\right)>0, T^{*}=T \cdot\left(r^{*}\right) \geqslant 0, w^{*}=w \cdot\left(r^{*}\right)>0$, удовлетворяющие оценке

$$
W \geqslant w^{*}>0, t \geqslant T^{*},\left|r-r^{*}\right| \leqslant \varepsilon^{*}, \forall \xi \in D^{\prime}{ }_{0}, t<\infty, a \in A .
$$

Пусть в $B=\left\{t \geqslant T^{*},\left|r-r^{*}\right| \leqslant \varepsilon^{*}, \xi \in D_{0}^{\prime}\right\}$ имеем неравенства

$-R_{1}\left(t, r^{*}, a\right) \leqslant\left. r^{*}\right|_{u=u^{\prime}}=R\left(t, \xi, a \mid u^{\prime}\right) \leqslant R_{2}\left(t, r^{*}, a\right) \quad\left(0 \leqslant R_{1}, R_{2} \subset C\right)$

с неотрицательными интегрируемыми на $\left[T^{*}, \infty\right)$ функциями $R_{\alpha}(\alpha=$ $=1,2)$. Обозначим через $T_{1}-, T_{2}^{-}, T_{1}{ }^{+}, T_{2}{ }^{+}$решения уравнений

$$
\begin{aligned}
& \int_{-T^{-}}^{0} R_{1}(t+\tau) d \tau=\varepsilon_{-}^{*}=\int_{-T^{-}}^{0} R_{2}(t+\tau) d \tau\left(T-\frac{\alpha}{\alpha}=T_{\alpha}^{-}\left(t, \varepsilon_{-}^{*}\right)>0, \alpha=1,2\right), \\
& \int_{0}^{T^{*}} R_{1}(t+\tau) d \tau=\int_{0}^{T^{*}} R_{2}(t+\tau) d \tau=\varepsilon_{-}^{*}=\varepsilon^{*}-\varepsilon_{*}^{*}>0 \quad\left(T_{\alpha}^{+}=T_{\alpha}^{+}\left(t, \varepsilon_{-}^{*}\right)\right),
\end{aligned}
$$

дающие оценки снизу левого, правого и полного отрезков времени включения $r *[\tau] \in B$ для начального $r_{*}[t]$ из $\left|r_{*}[t]-r^{*}\right| \leqslant \varepsilon *$ :

$$
\begin{aligned}
& \Delta t^{-} \geqslant \Delta t_{1}^{-}=\min \left\{t-T^{*}, T_{1}^{-}, T_{2}^{-}\right\} \equiv T^{-}\left(t, \varepsilon_{-}^{*}\right), T_{0}^{*}=T-+T^{+}, \\
& \Delta t^{+} \geqslant \Delta t_{2}^{+}=\min \left\{T_{1}^{+}, T_{2}^{+}\right\} \equiv T^{+}\left(t, \varepsilon_{-}^{*}\right), \Delta t=T_{0}^{*}\left(t, \varepsilon_{-}^{*}\right) .
\end{aligned}
$$

Пусть для любой последовательности $t_{k} \rightarrow \infty, 0<t_{k}<t_{k+1}$ ряд

$$
\begin{gathered}
S_{m}^{*}=\sum_{k=1}^{m} T_{0}^{*}\left(t_{k} \mid \varepsilon_{-}^{*}\right)\left(\varepsilon_{*}=\varepsilon_{*}\left(t_{k}\right) \rightarrow 0,0<\varepsilon_{-}^{*}=\varepsilon^{*}-\varepsilon_{*}, k, m \rightarrow \infty\right), \\
S^{*}=\lim S_{m}^{*}=+\infty(m \rightarrow \infty)
\end{gathered}
$$

расходится при $m \rightarrow \infty, \forall \varepsilon *$ и $\varepsilon^{*}>\varepsilon_{*} \geqslant 0$ в оценках (1.10), (1.11).

Л ем м а 1. Возможный регулятор $u^{\prime}(t, \xi, a)$ будет $x^{1}, x^{\prime}$-стабилизируюциим (1.1) для $t_{0}=$ fixconst $\geqslant 0,\left|x_{0}{ }^{1}\right| \leqslant \delta_{0}{ }^{1}, x_{0}^{(2)}$, если функции $V, u^{\prime}$ удовлетворяют условиям (1.5), (1.10), (1.11), (1.14) для $\forall a \in A$.

Доказ ательст в о. С учетом (1.5), (1.6) достаточно показать при условиях леммы отсутствие решений $\xi *[t]$ с $r$--компонентой для системы (1.11). Допустив противное, рассмотрим $r \cdot[t]$, для которой в силу (1.7) $-(1.9)$ есть $\tau_{k}{ }^{*} \rightarrow+\infty$ со свойствами

$$
\begin{aligned}
& r\left[\tau_{k}^{*}\right] \equiv r_{* k} \rightarrow r^{*}, d r_{*} / d \tau_{k}^{*} \rightarrow 0\left(0<r^{*} \leqslant H, \tau_{h}^{*}<\tau_{k+1}^{*}, k=\overline{1, \infty}\right), \\
& \forall \varepsilon_{*}>0 \exists T \cdot \leqslant \tau_{n}:\left|r_{*}\left[\tau_{n}\right]-r^{*}\right| \leqslant \varepsilon_{*}<\varepsilon^{*} \quad\left(\left\{\tau_{n}\right\} \subseteq\left\{\tau_{k}^{*}\right\}, n \rightarrow \infty\right) .
\end{aligned}
$$


Интегрируя вдоль $\xi *[t]$ при $u=u^{\prime}$ равенство $V^{\cdot}=-W$, с учетом $(1.10)-(1.15)$ имеем неравенства

$$
V\left[t_{0}\right]-V[\infty]=\int_{t_{0}}^{\infty} W[\tau] d \tau \geqslant w^{*} S^{*}=\infty\left(V\left[t_{0}\right] \geqslant V[\infty] \geqslant 0\right)
$$

в силу их противоречия $\{r *[t]\}=\varnothing$. Лемма доказана.

3 а меч ания. Для $r$-агрегированной предельной точки $r^{*}=H$ функция $T_{0}{ }^{*}\left(t, \varepsilon_{-}{ }^{*}\right)$ имеет вид $T_{1}{ }^{+}+T_{0}-\left(T_{0}-=\min \left\{t-T_{H^{*}}{ }^{*}, T_{2}{ }^{-}\right\}, T_{0}^{*}=\right.$ $\left.=T_{1}+\left(R_{2}=\infty\right), T_{0}-\left(R_{1}=\infty\right)\right)$. Если $P=\varnothing$ для $u^{\prime}$-произведенной $V$ вида (1.5), то $\varnothing=\{r *[t]\}$ и $u^{\prime}(t, \xi, a)-x^{1}, x^{\prime}$-стабилизирующий (1.1) регулятор. Условия леммы 1 ослабляются заменой на положительно рекуррентное, предельно $r$-стационарное множество $P_{0} \subseteq P$. Точка $p_{0}$ входит в $P_{0}=\left\{\left(\infty, r_{0}{ }^{*}\right)^{*}\right\}$, если для $(1.1)\left(u=u^{\prime}\right)$ существует последовательность $\tau_{k} \rightarrow+\infty$ со свойствами

$$
\left|R\left(\tau_{k}, \xi\left[\tau_{k}\right], a \mid u^{\prime}\right)\right| \stackrel{\downarrow}{-} 0, \quad r\left[\tau_{k}\right] \equiv\left|y^{\prime}\left[\tau_{k}\right]\right| \rightarrow r_{0}^{*} \in(0, H],
$$

где $0<r[t] \leqslant H \quad$ при $\left|y_{0}\right| \leqslant \delta^{0}, 0<\left|y_{0}^{\prime}\right| \leqslant \delta^{\prime 0} \leqslant \delta^{0}, t_{0} \geqslant 0, \gamma_{0} \in E^{n-l_{0}}$,

$$
f[t]=f\left(t, t_{0}, y_{0}, \gamma_{0}, a \mid u\right),\left(t_{0}, \gamma_{0}, a\right)^{*}=\text { fixconst. }
$$

Установим второй критерий $x^{1}, x^{\prime}$-стабилизации (1.1) при $u=u^{\prime}$. Примем, что функция $r[t]$ при $u=u^{\prime}, \xi \in D_{0}^{\prime}, t \geqslant 0, \forall a \in A$ удовлетворяет дифференциальным неравенствам

$$
\left|r^{\prime}\right| \equiv|R| \leqslant R_{1}(t, r, a)\left(0<R_{1} \subset C\left(B^{1}\right), \partial R_{1} / \partial r \subset C\left(B^{1}\right)\right),
$$

где $B^{1}=\{0 \leqslant t<\infty, 0<r \leqslant H\}, \forall a \in A$.

Пусть все $r_{1}-+\infty$-продолжаемые решения $r_{1}{ }^{*}=-R_{1}$ и верно

$$
\lim r_{1}[t]>0, t \rightarrow f \infty \quad\left(\forall t_{0}, r_{10} \in B^{1}, a \in A\right) .
$$

На $B^{1}$ для системы (1.4) при $u=u^{\prime}$ предположим свойства:

$$
-\left.V^{\cdot}\right|_{u=u^{\prime}} \equiv W \geqslant W_{1}(t, r, a) \geqslant 0\left(W_{1}, \partial W / \partial r \subset C\left(B^{1}\right), \forall a \in A\right),
$$

$$
\omega_{1} \equiv \partial W_{1} / \partial r \geqslant 0, t, r \in B^{1}, \Delta^{+} \omega\left|t^{0}, r^{0} \equiv \omega\right| t^{0}+\Delta t-\omega\left(t^{0}, r^{0}\right)>0(0<\Delta t \leqslant \varepsilon) \text {, }
$$

$$
\lim _{T \rightarrow+\infty} \int_{t_{0}}^{T} W_{1}\left(\tau, r_{1}[\tau], a\right) d \tau=\infty, r_{10}, t_{0} \in B^{1}, \forall a \in A\left(\forall t^{0}, r^{0} \in\left\{\omega_{1}=0\right\} \cap B^{1}\right) .
$$

Покажем, что $\xi_{1}[t] \equiv-1, r_{1}[t]-$ оптимальное решение задачи

$$
\begin{gathered}
r=\xi R_{1},-1 \leqslant \xi \leqslant 1, \xi \in C\left[t_{0}, T\right]\left(0 \leqslant t_{0}<T<\infty\right), \\
0<r[\mathrm{t}] \leqslant H=\text { fixconst, } t_{0} \leqslant t<T \quad\left(r_{0}=r\left[t_{0}\right]>0,0<r[T] \leqslant H\right), \\
I_{1}\left(t_{0}, r_{0}, T, a \mid \xi\right)=\int_{t_{0}}^{T} W_{1}(\tau, r[\tau], a) d \tau \rightarrow \min _{\xi}=I_{1}\left(\xi^{0}\right) .
\end{gathered}
$$

Из (1.17), (1.20) и $\omega_{1} \geqslant 0$ находим $r_{1}[t] \leqslant r[t \mid \xi], I_{1}\left(\xi_{1}\right) \leqslant I_{1}(\xi)$ $\left(t \in\left[t_{0}, T\right], \xi_{1} \equiv-1, \forall \xi[t] \geqslant \xi_{1}[t], \xi \in C\right)-$ оптимальность $\xi_{1}$. Для (1.20) имеем необходимые условия

$$
\begin{gathered}
H_{1}\left(t, r_{1}, p_{1}, \xi_{1}, a\right) \leqslant H_{1}\left(t, r_{1}, p_{1}, \xi, a\right), \\
p_{1} \xi_{1}=\min p_{1} \xi=-p_{1}<0\left(H_{1}=W_{1} f p_{1} \xi R_{1}\right),
\end{gathered}
$$$$
p_{1}=\left(\partial R_{1} / \partial r_{1}\right) p_{1}-\partial W_{1} / \partial r_{1}, \quad p_{1}[T]=0\left(p_{1}=\int_{t}^{T} \Phi_{1}(t, \tau) \omega_{1}^{1}(\tau) d \tau, \quad t_{0} \leqslant t \leqslant T\right),
$$ 


$$
\Phi_{1}=\exp \left[\int_{\tau}^{t} \partial R_{1} / \partial r_{1} d \xi\right], r_{1}=r_{1}[\xi], \omega_{1}^{1}=\partial W_{1} / \partial r_{1}=\omega_{1}^{1}\left(\tau, r_{1}[\tau], a\right) \geqslant 0,
$$

где $p_{1}>0$ на $\left[t_{0}, T\right)$ в силу локальной неинвариантности $\left\{\omega_{1}=0\right\}$ вдоль $r_{1}[t]$ при (1.19). Тогда согласно (1.20) и $\left|r_{*}\right| \leqslant R_{11}\left(r_{*}\right)$ аналогично лемме 1 получаем из $I_{1}\left(r_{1}\right) \leqslant I_{1}\left(r_{*}\right)$ доказательство леммы 2 .

Л е м м 2. Регулятор и' дает $x^{1}, x^{\prime}$-стабилизацию (1.1), если функции $V, u^{\prime}$ удовлетворяют условиям (1.5), (1.17)-(1.19).

2. Зададим $u^{+}\left(t, \xi, a \mid S^{+}, \lambda^{+}\right) \in U^{\prime}$ потенциалом $S^{+}(t, \xi, a) \geqslant 0$ и штрафом $\lambda^{+}(t, \xi, a, u) \geqslant 0$ задачи обращения аналитического конструирования:

$$
\begin{gathered}
J^{+}\left(t, \xi, a \mid u, \lambda^{+}\right)= \\
=\int_{t}^{t^{\prime}} \lambda^{+} f_{0} d \tau\left(S^{+}, \lambda^{+} \in C\left(D_{0} \times \Omega\right), \lambda^{+}, S^{+} \in C_{1}\left(D^{\prime}{ }_{0} \times \Omega\right), \forall a \in A\right), \\
J^{+}\left(u, \lambda^{+}\right) \rightarrow \underset{u}{\min }= \\
=S^{+}\left(t^{1}=\min t^{*}: y^{\prime}\left[t^{*}\right]=0,0<\left|y^{\prime}\right| \leqslant H, 0<|y| \leqslant H, t<t^{1}\right) .
\end{gathered}
$$

Пусть для $S^{+}, \lambda^{+} \exists V^{+}, u^{+}$, удовлетворяющие лемме 1 или 2 , причем $H^{+}(t, \xi, u, a)>H^{+}\left(t, \xi, u^{+}, a\right), u^{+} \neq u \in \Omega\left(H^{+}=\lambda+f_{0} f \partial S+/ \partial t+f \cdot \partial S+/ \partial \xi\right)$,

$$
H^{+}\left(t, \xi, u^{+}, a \mid S^{+}, \lambda^{+}\left(u^{+}\right)\right) \equiv 0,
$$

$$
t \geqslant 0, \xi \in D_{0}^{\prime}, \forall a \in A\left(H\left(t, \xi, u, a \mid S^{+}, \lambda^{+}\right) \equiv H^{+}\right),
$$

$\left.S^{+}\right|_{y^{\prime}=0}=0, u^{+}=u_{+}^{\prime}(t, \xi, a) \in C\left(t \geqslant 0, \xi \in D_{0}^{\prime}\right), \lambda^{+}, S^{+} \in C_{1}\left(t \geqslant 0, D_{0}^{\prime} \times \Omega\right)$,

$\forall a \in A$.

Тогда имеем $x^{1}, x^{\prime}$-стабилизацию $\quad(1.1) \quad\left(u=u_{+}(t, x, a)=\right.$ $\left.=u_{+}^{\prime}[t, \xi(t, x, a), a]\right)$, существование, единственность и непрерывность $u^{+}$, строго минимизирующего $H^{+}$в $\Omega$.

Т еорем а. Регулятор $u_{+}^{\prime}$ оптимально по (2.1) $y, y^{\prime}$-стабилизирует (1.4) в области (1.6) для значений $t_{0}, \xi_{0} \in C^{0}=\left\{t_{0} \geqslant 0, \quad \xi_{0} \in D_{0}^{\prime}\right.$, $\left.\left|y_{0}\right| \leqslant \delta^{0}\right\} \quad(a \in A)$, если для $S^{+}, \lambda^{+}, u_{+}^{\prime}$ выполнены условия (2.2) $и$ леммы 1 или 2.

Доказ ательств о. Из. выполнения условий леммы 1 или 2 следует $y, y^{\prime}$-стабилизация (1.4) при $u=u_{+}^{\prime}(t, \xi, a)$. Интеграцией неравенств $(2.2)$ из краевого условия на $S^{+}(t, \xi, a)$ для $u_{+}^{\prime}$ и стабилизирующего $u$. $\not u_{+}^{\prime}$ получаем неравенства

$$
S^{+}(t, \xi, a)=\int_{t}^{t^{1}} \lambda^{+}\left(u_{+}^{\prime}\right) f_{0}\left(u_{+}^{\prime}\right) d \tau<J+\left(u_{*}, \lambda\left(u_{*}\right)\right)=\int_{t}^{t \dot{t}^{\prime}} \lambda^{+}\left(u_{*}\right) f\left(u_{*}\right) d \tau,
$$

означающие единственность минимизирующего (2.1) регулятора $u_{+}^{\prime}$.

Теорема доказана. Поскольку $\lambda^{+} \not \equiv 1, u_{+}^{\prime}-$ субоптимальный регулятор $\left[{ }^{3}\right]$ системы (1.4) на $C^{0}$.

Замкнем систему (1.1), (1.2) с плоскостью цели $Q$ субоптимальным, стабилизирующим ее локально при $\forall a \in A$ регулятором $u_{+}(t, x, a)$ :

$$
\begin{gathered}
x=\Phi(t, x, a), t, x \in\left\{t \geqslant 0, x\left(D_{0}^{\prime}\right), \varrho \leqslant H\right\}(x=x(t, \xi, a), a \in A), \\
a=0, Q=\left\{x \mid x^{\prime}=0\right\}\left(u_{+}=u_{+}^{\prime}(t, \xi(t, x, a), a), \Phi=F\left(t, x, u_{+}\right)\right), \\
J_{+}\left(t_{0}, x_{0}, a\right)= \\
=J\left(t_{0}, x_{0} \mid u_{+}\right)=\int_{t_{0}}^{t^{\prime}} \Phi_{0}(\tau, x, a) d \tau\left(\Phi_{0}=F_{0}\left(\tau, x, u_{+}\right), t^{1}=T_{1}\left(t_{0}, x_{0}, a\right) \neq t_{1}\right),
\end{gathered}
$$


$x_{0}=x\left[t_{0}\right]=x\left(t_{0}, \xi_{0}, a\right), x_{1}^{\prime}=x^{\prime}\left[t^{1}\right]=0\left(t^{1}=\min t^{*}: \lim x^{\prime}[t]=0, \quad t \rightarrow t^{*}-0\right)$, $t_{0}=$ fixconst $\geqslant 0, \xi_{0} \in D_{0}^{\prime},\left|y_{0}\right| \leqslant \delta^{0},\left|y_{0}^{\prime}\right| \leqslant \delta_{0}^{\prime 0}\left(t^{1}=\min t^{*}: \lim y^{\prime}[t]=\right.$

$\left.=0, t \rightarrow t^{*}-0\right)$.

Для (2.3) рассмотрим минимизацию $J_{+}$по $a$ при фиксированных $t_{0}, x_{0}$ и условиях $\varphi_{\alpha}(x) \equiv x_{\alpha}^{\prime}=0 \quad\left(\alpha=\overline{1, m_{0}} \leqslant m_{1} \leqslant n\right)$, что эквивалентно выбору $u_{+}{ }^{0}-$ наилучшего по (1.2) субоптимального регулятора (1.1), порожденного $\lambda^{+}, S^{+}$из классов $(2.1),(2.2)$. Введем гамильтониан [ $\left.{ }^{4,5}\right]$ и обозначения

$$
\begin{gathered}
G(t, z, \psi)=v_{0} \Phi_{0}+\psi \cdot Z, z=\left(x^{*}, a^{*}\right)^{*}, Z=\left(\Phi^{*}, 0\right)^{*}, \psi=\left(\psi_{k}\right)^{*}, \\
\psi=\left(p^{*}, \Theta^{*}\right)^{*}, p=\left(p_{i}\right)^{*}, \Theta=\left(\Theta_{m}\right)^{*} \quad(i=\overline{1, n}, m=\overline{1, N,} k=\overline{1, n+N) .}
\end{gathered}
$$

Из (2.3), (2.4) имеем необходимые условия оптимальности

$p_{\alpha}\left(T_{1}^{0}\right)=v_{\alpha}, \psi_{j}\left(T_{1}^{0}\right)=0\left(\psi\left(T_{1}\right)=v_{\alpha} \partial \varphi_{\alpha} / \partial z \mid t=T_{1}^{0}, j=m_{0}+1, n+N\right)$,

$$
\begin{gathered}
v_{0} \Phi_{0}\left(T_{1}^{0}, x^{0}\left[T_{1}^{0}\right], a^{0}\right)+v_{\alpha} \Phi_{\alpha}\left(T_{1}^{0}, x^{0}\left[T_{1}^{0}\right], a^{0}\right)=0\left(\left.G\right|_{t=T_{1}{ }^{0}=0,} \alpha=\overline{1, m_{0}}\right), \\
\Theta\left[t_{0}\right]=\Theta\left[T_{0}^{1}\right]=\int_{T_{1}^{0}}^{t_{0}}\left(\partial g / \partial a^{0} \mid x=x^{0}, p=p^{0}\right) d \tau=0 \quad\left(\Theta=-\partial G / \partial a^{0}\right),
\end{gathered}
$$

где $x^{0}[t], p^{0}[t], T_{1}^{0}=T_{1}\left(t_{0}, x_{0}, a^{0}\right)$ - соответствующие $a^{0}$ траектория, импульс и момент примыкания (2.3), для которых имеем

$$
\begin{gathered}
d x^{0} / d t=\Phi\left(t, x^{0}, a^{0}\right), d p^{0} / d t=-\partial g / \partial x^{0}, \Theta=-\partial g / \partial a^{0}\left(a^{0}=\text { opt } a\right), \\
g(t, x, p, a)=v_{0} \Phi_{0}+p \cdot \Phi, x^{0}\left[t_{0}\right]=x_{0}, x^{\prime 0}\left[T_{1}^{0}\right]=0, t_{0}=\text { fixconst } \geqslant 0 .
\end{gathered}
$$

При этом $\left(v_{0}, v_{\alpha}\right)^{*} \neq 0,0 \neq\left(v_{0}, \psi^{*}\right)^{*} \in C\left[t_{0}, T_{1}^{0}\right) \quad\left(\alpha=\overline{1, m_{0}}\right)$.

3. Пр и ме р. Рассмотрим субоптимальный синтез системы

$$
x=f(x, u), f(0,0), f \in C_{1}\left(E^{n} \times \Omega\right), x=\left(x_{i}\right)^{*}, x^{1}=\left(x_{j}\right)^{*}, x^{(2)}=\left(x_{\sigma}\right)^{*},
$$

$$
\begin{gathered}
d x^{1} / d t=f^{1}(x, u), i=\overline{1, n}, j=\overline{1, n_{1}} \leqslant n, \sigma=\overline{n_{1}+1, n,} \\
u=\left(u_{s}\right)^{*}, \Omega=\left\{u \mid 0 \leqslant v(u) \leqslant v_{0}\right\}, v(\alpha u)=\alpha v(u), \\
\alpha \geqslant 0\left(v_{0}=\text { const, } s=\overline{1, r} \leqslant n\right), \\
v(u)>v(0)=0, u \neq 0, v\left(u_{2}\right)-v\left(u_{1}\right)>\left(u_{2}-u_{1}\right) \partial v / \partial u_{1} \\
\left(\alpha_{\delta} u_{\delta} \neq 0 \alpha_{\delta}^{2} \neq 0, \delta=1,2\right), \\
v \in C\left(E^{r}\right), v \in C_{1}\left(E^{r} \backslash 0\right), Q=\left\{x \mid x^{1}=0\right\}, t_{1}=\min t_{*}: \lim x^{1}[t]=0, \\
t \rightarrow t *-0,
\end{gathered}
$$

$$
J=\int_{0}^{t_{1}} f_{0}(x, v) d \tau \rightarrow \min _{u \in \Omega}\left(0<f_{0} \in C\left(E^{n} \times \Omega\right), f_{0} \in C_{1}\left(E^{n} \backslash Q \times \Omega \backslash 0\right)\right)
$$

с агрегатом - производящей функцией $S^{+} \equiv y(x, a) \equiv a h\left(x^{1}\right)$ вида

$$
\begin{gathered}
y=a h\left(x^{1}\right), h\left(x^{1}\right)>h(0)=0, \partial h / \partial x^{1} \neq 0\left(x^{1} \in\left\{x \mid 0<h\left(x^{1}\right) \leqslant h^{0}\right),\right. \\
0<\mathrm{const}=a_{1}<a<a_{2}=\mathrm{const} \leqslant \infty, h^{0} \leqslant \sup h\left(E^{n_{1}}\right), h \in C_{1}\left(E^{n_{1}} / 0\right), \\
h \in C\left(E^{n_{1}}\right)
\end{gathered}
$$

для вспомогательного критерия $J^{+}$с множителем штрафа $\lambda^{+}(h, a)$ 


$$
J^{+}=\int_{0}^{t^{1}} \lambda^{+} f_{0} d \tau \rightarrow S^{+}=\min _{u \in \Omega}, 0<\lambda^{+} \subset C_{1}\left(0<h \leqslant h^{0}, a_{1}<a<a_{2}\right) .
$$

Введем гамильтониан $G$ вспомогательной задачи и обозначения

$$
\begin{gathered}
G=\lambda f_{0}(x, v)+a g \cdot f^{1}\left(g \equiv \partial h / \partial x^{1}, u=v \alpha, v(\alpha)=1,0 \leqslant v \leqslant v_{0}\right), \\
k(x, v) \equiv g \cdot f^{1}\left(x, v \alpha_{+}\right) \equiv \min _{\alpha}\left[g \cdot f^{1}(x, v \alpha)\right], \alpha_{+}(x, v)=\operatorname{argmin}[\cdot], \\
H=\lambda f_{0}(x, v)+a k(x, v), P=P(v, x, a, \lambda)=\underset{a \leqslant v \leqslant v_{0}}{\max }(v \cdot v-H), \\
v=\partial H / \partial v, v=\partial P / \partial v, P\left(0, x, a, \lambda^{+}\right)=0, v_{0}=0, v^{+}=\partial P / \partial v_{0} \mid \lambda=\lambda^{+} .
\end{gathered}
$$

Здесь для $x^{1}$-стабилизации и простоты приняты условия

$$
k\left(x, v^{+}\right) \leqslant-w_{1}\left(x^{1}, a\right)\left(0<h \leqslant h^{0}, a \in\left(a_{1}, a_{2}\right), 0<w_{1} \subset C\left(0<h \leqslant h^{0}\right)\right),
$$

$\partial^{2} H / \partial v^{2}>0,0<v \leqslant v_{0}, \partial H /\left.\partial v\right|_{v=0}<0, \partial H / \partial v_{0} \geqslant 0$,

$$
\lambda^{+}=\lambda^{+}(h, a): P\left(0, x, a, \lambda^{+}\right) \equiv 0\left(0<h \leqslant h^{0}, a \in\left(a_{1}, a_{2}\right)\right) .
$$

Тогда $y$ и оптимальный по (3.3) $u^{+}$удовлетворяют неравенствам $\left.y^{\cdot}\right|_{u=u^{*}}=a k\left(x, v^{+}\right) \leqslant-a w_{1}\left(x^{1}, a\right)\left(x \in\left\{0<h\left(x^{1}\right) \leqslant h^{0}=\right.\right.$ const $\left.\}, \forall a \in A_{2}^{1}\right)$,

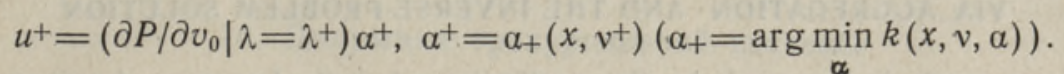

$H(v), P(v)$ - строго выпуклы. $G$ имеет в $v^{+}$строгий нулевой стационарный по $v$ минимум при $\lambda=\lambda^{+}, \lambda^{+}$не зависит явно от $x$. По теореме п. 2 $u^{+}-$субоптимальный регулятор (3.1), ибо в силу (3.2), (3.4)-(3.7) $u^{+}(x, a) \quad x^{1}$-стабилизирует (3.1) и минимизирует (3.3) для $x_{0}{ }^{1} \in$ $\in\left\{0 \neq h\left(x_{0}^{1}\right)=h_{0} \leqslant h^{0}\right\}$. Из (3.2), (3.4)-(3.6), замыкая (3.1) регулятором (3.7), получаем

$$
J\left[u^{0}\right]=\min _{u \in \Omega} J\left(x_{0} \mid u\right) \leqslant J_{+}(a) \equiv \int_{0}^{h_{0}} \Phi(h, a) d h\left(\Phi^{+} \equiv a \lambda^{+}>0,0<h \leqslant h^{0}\right) .
$$

Пусть задача минимизации $J_{+}(a)$ на $\left(a_{1}, a_{2}\right)$ имеет смысл

$$
\lim \int_{\varepsilon}^{h_{0}} a \lambda^{+^{-1}}(\xi, a) d \xi<+\infty \quad(\varepsilon \rightarrow 0+), \exists a^{+}=\arg \min J_{+}\left(a \in\left(a_{1}, a_{2}\right)\right) .
$$

Условия (2.5) определения оптимального для (3.8) числа $a^{0}$ упрощаются

$$
\int_{0}^{h_{0}}\left(\lambda^{+}-a^{0} \partial \lambda^{+} / \partial a^{0}\right) \lambda^{+^{-2}} d \xi=0\left(\lambda^{+}: P\left(0, x, a, \lambda^{+}\right) \equiv 0\right) .
$$

Сравнением $J_{+}\left(a^{0}\right)$ для решений (3.9) находим $a^{+}$и оценку $J\left(u^{Q}\right) \leqslant$ $\leqslant J_{+}\left(h_{0}, a^{+}\right)$.

\section{ЛИТЕРА Т Р А}

1. Р у м я нц е в В. В., Прикл. мат. и мех., 34, вып. 3, 440-456 (1970).

2. A o ki, M., In: Optimization methods for large-scale systems, 5 Aggregation, New York, McGraw Hill Book Company, 1971, p. 191-232.

3. К е й с И., Изв. АН ЭССР, Физ. Матем., 27, № 3, 274-288 (1978). 
4. Болтянски й В. Г., Математические методы оптимального управления, M., «Наука», 1966, с. 295-300.

5. Моисеев Н. Н., В кн:: Численные методы в теории оптимальных систем, М., «Наука», 1971, с. $32-34$.

Ннститут кибернетики

Академии наук Эстонской ССР

Поступила в редакцию 29/VI 1978

I. KEIS

\section{DUNAAMILISTE SUSTEEMIDE SUBOPTIMAALNE SUNTEES POORD- JA AGREGEERIMISMEETODIL}

On esitatud kaks dünaamilise reguleeritava süsteemi stabiliseerimise kriteeriumi, tõestatud suboptimaalse regulaatori $u(t, x, a)$ sünteesi teoreem ning leitud optimaalse agregeerimisvektori määramise tingimused.

\section{KEIS}

\section{ON SUBOPTIMAL STABILIZATION METHOD FOR DYNAMIC SYSTEMS VIA AGGREGATION AND THE INVERSE-PROBLEM SOLUTION}

A new approach is proposed to the application of both Lyapunov functions and $y(t, x, a)$-informative variables to suboptimal aggregation of large-scale systems.

As a result, new relaxed controllability and stability conditions are established in the lemmas 1 and 2 for non-stationary non-linear controllable large-scale dynamic systems.

A theorem on the synthesis of efficient suboptimal controls $u(t, x, a)$, introduced via generating potential of the inverse problem and aggregation variables $y(t, x, a)$, is proposed.

Necessary conditions for the choice of the aggregation vector $a$, minimizing the performance index, are obtained.

The suboptimal control policy is derived by applying this approach for a special class of $y$-autonomous systems. 